\title{
LA RIPPET Y LOS APORTES AL CONOCIMIENTO Y LA COOPERACIÓN ACADÉMICA. \\ El camino recorrido entre los encuentros de la ciudad de La Plata, Argentina y Manizales, Colombia.
}

\author{
RIPPET AND CONTRIBUTIONS TO KNOWLEDGE AND \\ ACADEMIC COOPERATION.
}

The achievements between the meetings held in La Plata, Argentina and Manizales, Colombia.

José Blanes Jiménez. ${ }^{1}$

BOLIVIA

Palabras Clave: Cooperación académica; calidad; visibilidad; formación profesional; diálogo universidad sociedad; desarrollo territorial; investigación docencia.

Keywords: Academic cooperation, quality, visibility, professional training, university and society dialog; territorial development; research and teaching.

\section{Resumen}

En su VIII encuentro internacional la RIPPET reflexiona sobre las formas de organización que le permitan responder las principales preguntas y retos del momento en la región marcada por dos fenómenos: la masificación de la formación superior y las brechas crecientes entre países e instituciones. Cómo agregar valor a la calidad de los programas y de las respuestas, cómo concretar el compromiso con la comunidad, cómo dar visibilidad a los programas de formación y capacitación profesional, cómo organizar comunidades de aprendizaje para crear espacios de cooperación. La RIPPET analiza el tipo de institucionalidad que le permita agregar valor a los programas con base en criterios de cooperación y colaboración, maximizando los recursos de las instituciones y programas que componen al Red.

\section{Abstract}

The VIII International meeting of RIPPET analyzes the forms that an organization responds to the recent principal questions and challenges in the region. It focuses especially on two phenomena: the increasing growth of higher educational training programs and the increasing gaps between countries and institutions. Some of the questions are the following: "How can value be added

1.Secretario General Red I beroamerican a d e Postgrados en Planeación y Políticas Territoriales, RIPPET. Director del Centro Boliviano de Estudios Multidisciplinarios, CEBEM. Bolivia. 
to the quality of programs and academic opportunities?", "How to meet the commitment to the community?", "How to provide visibility to educational programs and professional training?", and "How to organize learning communities in order to create cooperative spaces?" RIPPET, through the analysis of the institutional framework, adds value to programs based on criteria for cooperation and collaboration, which increases the resources of the institutions and programs comprising the network.

\section{LA EDUCACIÓN SUPERIOR, RETOS DEL MOMENTO ACTUAL.}

Los temas que hoy nos preocupan a quienes hemos llegado hasta aquí no son nuevos y han llamado la atención de muchas instituciones de educación superior en la Región y fuera de ella. Muchos de estos temas son compartidos, por gran parte de las redes conformadas por su interés en la relación entre la Universidad la comunidad, los Estados y la cooperación para el desarrollo. Es obvio que esta relación interesa cada vez más a los miembros de RIPPET representantes de programas de postgrado en temas territoriales de la región.

Hoy día existen muchas experiencias en la conformación de redes y nos preguntamos cuánto es lo que agrega en valor la RIPPET y si es una más en un bosque cada vez más frondoso de experiencias. Experiencias de asociación como Global Alliance, Vertebralcue, entre otras muchas, nos pueden servir para profundizar sobre el posible aporte de RIPPET.

- Global Alliance for Community-engaged Research (GACER) es una red, creada en mayo de 2008 que cuenta con la representación de 14 países. En su declaración firmada por más de 600 delegados plantea que la red es una oportunidad para aprovechar las fortalezas existentes entre varias redes y avanzar en la aplicación del desarrollo del conocimiento en alianzas entre la universidad y la sociedad en pro del desarrollo democrático, para un cambio social y ambiental. Se propone facilitar movimientos activistas mediante la emergencia de redes a nivel mundial para el fortalecimiento de las organizaciones de base y la presión por la solución de problemas tan complejos como la injusticia, la violencia, la pobreza, el cambio climático y la salud en todo el mundo. www.communityresearchcanada.ca

Las instituciones que componen esta alianza se insertan en un movimiento muy amplio de redes temáticas en casi todo el mundo, unidas en el intento de vincular las actividades académicas a los problemas cotidianos, particularmente la ciudadanía, la democracia, la gobernabilidad, la lucha contra la pobreza. Se trata de instancias universitarias relativamente desconcentradas. Presenta sus ideas y propuestas en foros internacionales globales como la UNESCO WCHE 2009 y otros regionales en Canadá, Malasia, UK. (GACER, 2009)

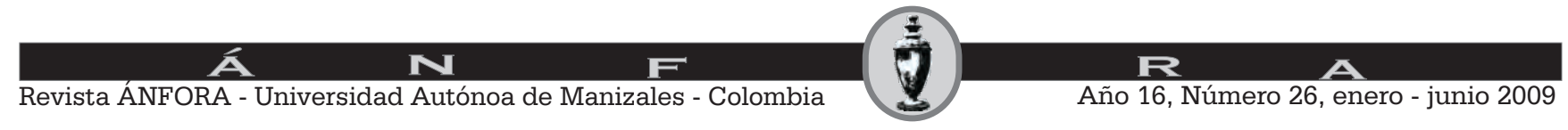


- Vertebralcue (ALFA III) es un proyecto de grandes dimensiones tanto en términos económicos como de cobertura institucional. La Comisión Europea asignó 3 millones de euros de los 19 disponibles para los 17 proyectos ALFA III. Vertebralcue abarca 17 países y 32 instituciones de Educación Superior: 25 instituciones de países latinoamericanos (Argentina, Chile, Brasil, Bolivia, Uruguay, Paraguay, México, Nicaragua, Costa Rica, Perú, Ecuador, Colombia, Venezuela) 4 europeos (Italia, España, Portugal y Austria). Entre las 32 instituciones se incluyen universidades, asociaciones de universidades (como ASCUN de Colombia, que representa otras 82 universidades, y ANUIES de México, que representa otras 150 universidades), centros de estudio e investigación y la red FLACSO de Facultades Latinoamericanas de Ciencias Sociales (representada en el proyecto por sus Sedes de México, Ecuador y Argentina). A las 32 instituciones Partners se agrega un Partner asociado, CONEAU, del Ministerio de Educación de Argentina, y otros posibles futuros Partners asociados que, desde su constitución, han expresado la intención de adherirse a la red.

http://www.ba.unibo.it/BuenosAires/Institucional/Sede/vertebralcue.htm

Ambos proyectos que funcionan en formato de red, muestran dos formas diferentes de acción en torno a problemáticas muy similares. La primera red se coloca en la perspectiva de asociación universidad - comunidad y la segunda mira preferentemente hacia adentro del mundo académico, aunque incluye también una atención importante a otros stakeholders como son las comunidades de base o los gobiernos. La primera mira al desarrollo de un activismo mundial y la segunda mira al mundo de la cooperación académica en el marco de a la relación entre Europa y América Latina y Caribe. La primera se concentra en el relevamiento de experiencias exitosas que resalten el compromiso de las Instituciones de Educación Superior (IES), mientras que la segunda se concentra en el análisis de formas y modelos de cooperación entre IES de Europa y LAC.

Una aproximación a la creciente formación de redes universitarias latinoamericanas y caribeñas ofrece algunas líneas claves del panorama general, trazos básicos sobre la naturaleza y funcionamiento de los proyectos en red. $^{2}$ En dichos análisis las redes muestran las principales áreas de acción y las estrategias más recorridas, como también las deficiencias para alcanzar sus objetivos. Hay diferencias entre ellas, aunque los temas de acción son similares así como sus estructuras.

Las principales redes latinoamericanas de educación superior se orientan a promover:

§ la colaboración y estrechar lazos entre los miembros de la red

§ la excelencia académica y mejorar la gestión

$\S$ la movilidad de estudiantes, profesores e investigadores

$\S \quad$ la integración regional
2 Redes activas referidas en el sitio web de IESALC UNESCO http://www.iesalc.unesco.org.v e/index.php?option=com_cont ent\&view=article\&id=47\&ltemi $d=119$ \&lang=es. Otras búsquedas actualizadas en Gil y Obaya 2009 arrojan más información al respecto.

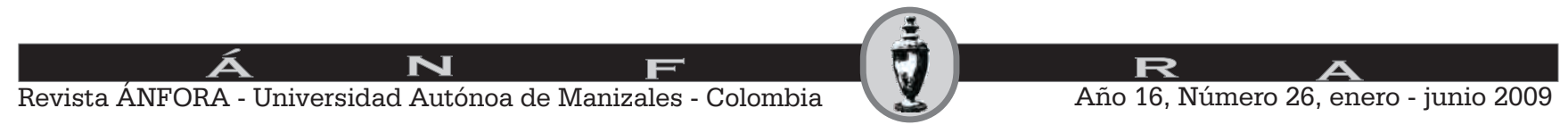


§ proyectos de investigación conjuntos

$\S$ proyectos de enseñanza conjuntos

§ colaboración con actores externos a la red

$\S$ el desarrollo sostenible y contribuir a la solución de problemas regionales

$\S$ fortalecer la interacción con la sociedad $^{3}$

$\S$ Intercambiar información ${ }^{4}$

§ armonizar los sistemas de información

En su segunda Conferencia Mundial sobre Educación superior la UNESCO 2009 llama la atención sobre el momento actual, en el que "nunca antes como ahora en la historia ha sido más importante invertir en la educación superior como una fuerza importante en la construcción de una sociedad del conocimiento y la diversidad y promover la investigación, la innovación y la creatividad."

Con toda la importancia de su formalidad la declaración de la UNESCO WCHE 2009 llama a aprovechar mecanismos de educación abierta y a distancia, y a la utilización de las TIC como oportunidades para "ampliar el acceso a una educación de calidad, especialmente cuando los recursos educativos abiertos son fácilmente compartidos por muchos países e instituciones de educación superior". Con el fin de garantizar que la introducción de las TIC agregue valor sugiere que "las instituciones y los gobiernos deberían trabajar juntos para poner en común experiencias, desarrollar políticas y fortalecer la infraestructura, especialmente el ancho de banda".

Las "Instituciones de educación superior de todo el mundo tienen una responsabilidad social para ayudar a salvar la brecha de desarrollo mediante el aumento de la transferencia de conocimientos a través de las fronteras, especialmente hacia los países en desarrollo, y trabajar para encontrar soluciones comunes para fomentar la circulación de cerebros y "aliviar los efectos negativos de la fuga de cerebros". "Redes universitarias internacionales y asociaciones son parte de esta solución y ayudan a mejorar el entendimiento mutuo y una cultura de paz".

“ Las asociaciones para la investigación y los intercambios de estudiantes y personal deben promover la cooperación internacional. En el fomento de una base más amplia y equilibrada la movilidad académica debe integrarse en los mecanismos que garanticen una verdadera colaboración multilateral y multicultural".

"La mayor cooperación regional es conveniente, en ámbitos tales como el reconocimiento de las calificaciones, la garantía de la calidad, la gobernanza, y la investigación y la innovación. La educación superior debe reflejar los planos internacional, regional y nacional en la enseñanza y la investigación. (WCHE 2009) $)^{5}$
3 Consecuentemente con el bajo nivel de prioridad asignado a este objetivo, fueron pocas las actividades específicamente orientadas a fomentar la interacción de las redes o las universidades con la sociedad. Sin embargo merecen destacarse el "monitor latinoamericano sobre cultura juvenil" en las universidades (AUSJAL) y el "Sistema Centroamericano de relación UniversidadSociedad" SICAUS) destinado a fortalecer la vinculación UNIVERSIDAD-SOCIEDAD e impulsar el enlace entre las universidades miembros de la Confederación Universitaria Centroamericana y los diferentes sectores de la sociedad centroamericana, para mejorar la calidad y la diversidad de la oferta de servicios y estudios de postgrado". El CSUCA cuenta también con el proyecto PUEDES que, a su vez, está integrado por 5 proyectos de diversa índole orientados a relacionar universidad y empresa con el objetivo de favorecer el desarrollo sustentable.

4 En algunos casos hay publicaciones de newsletters cuya frecuencia es irregular (AUALCPI, Gazeta UDUAL, ACTI), en otros hay "boletines informativos" que aparecen publicados en el mismo portal web (como en el caso de CSUCA). Sólo en dos casos se observó el desarrollo de sistemas de información y directorios sobre la organización, características académicas y organizativas de las instituciones que forman partes de las redes (UDUAL y UNAMAZ). 


\section{LA SITUACIÓN DE AMÉRICA LATINA}

En materia de educación superior América Latina es un continente donde las diferencias y las brechas entre países, así como entre las instituciones, son de las más altas del mundo. En América Latina tenemos todo lo mejor y todo lo peor. Las brechas se manifiestan entre universidades públicas y privadas, así como al interior de cada uno de estas dos categorías, pero sobre todo resaltan las diferencias entre países.

En el continente hemos presenciado también en estos últimos diez años, la emergencia de importantes programas de cooperación entre las instituciones de educación superior de América Latina, muchos de los cuales han operado entre países y entre continentes. El objetivo central explícito de éstos se orienta a la mejora de la calidad, la instalación de sistemas de acreditación que faciliten el movimiento de profesionales y estudiantes entre países, entre otros objetivos.

Estos programas han aumentado exponencialmente y se ha acumulado experiencia en una cantidad importante de instituciones. Pero al mismo tiempo y de la mano de la masificación de la educación superior y de la mercantilización de la educación universitaria, una gran cantidad de instituciones y por consiguiente de estudiantes, han quedado fuera de estas mejoras y aislados de estos aprendizajes que se han extendido por la región.

Sin embargo, hay razones para pensar que en ALC, con todos estos programas, no se alcanzan las soluciones a los problemas que enfrenta la sociedad en su relación con el uso del conocimiento. Persiste aún el desencuentro entre el sistema educativo y la realidad social, económica y política. Generalmente estos programas parten desde adentro y resaltan los problemas del sistema educativo, del aula, de los recursos educativos, pero no se enfocan los problemas de la sociedad frente al sistema educativo. El programa de cooperación termina siendo un tema del gremio de la educación superior. En este desencuentro el centro de educación superior es el sistema y no el profesional, vínculo real entre la educación y la aplicación del conocimiento. El profesional y su praxis no es considerado como el sujeto principal del sistema educativo; no es un actor de la producción de conocimiento que dialogue con el sistema. Finalmente las buenas prácticas, que existen en abundancia, quedan como acciones aisladas y no llegan a consolidar el conocimiento alcanzado, quebrándose el aprendizaje colectivo.

Tres temas centrales resaltan en estas experiencias:

1) La falta de visibilidad de las experiencias exitosas.

Este es un problema especialmente importante ya hay una gran cantidad de valiosos aprendizajes que podrían servir para acortar las brechas entre países e instituciones. Esta visibilidad se pierde además por la dificultad para la

5 http://www.unesco.org/fileadm in/MULTIMEDIA/HQ/ED/ED/pdf/ WCHE_2009/FINAL\%20COMM UNIQUE\%20WCHE\%202009.pdf

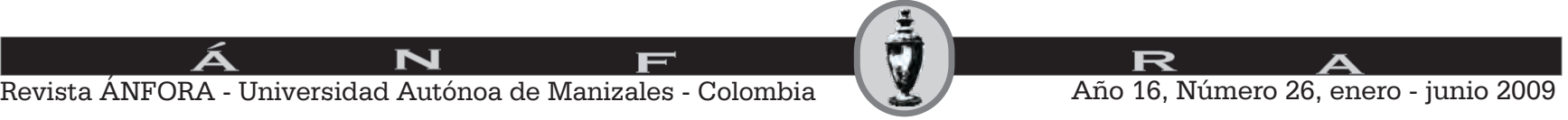


sistematización de un conjunto masificado de acciones; las formas de cooperación detectadas, además de ser masivas, son dispersas, puntuales, esporádicas, erráticas y espasmódicas.

2) El profesional es un actor secundario o ausente.

La insuficiente consideración del profesional egresado, principal vínculo entre el sistema y la realidad, quien desaparece una vez conseguido el titulo académico. Incluso se pierde el contacto con los colegios profesionales, los que se convierten en gremios para defender sus intereses corporativos. Se pierde contacto con este eslabón y la formación continua queda como un buen deseo en la mayor parte de los mejores casos.

3) La ausencia de espacios temáticos de acción.

La ausencia de un espacio común de acción, por mantenerse en el tema central el sistema mismo impide una acción sostenida, ordenada, sistemática, evaluable, de conocimiento acumulado en áreas especificas. El encuentro entre el sistema universitario y la realidad no se organiza en torno a programas de acción y de políticas a través de la investigación o de la formación de recursos o de proposición de políticas.

En el marco de este encuentro se pueden distinguir tres grandes desafíos relacionados con estos temas:

1) Estrechar la brecha entre los dos mundos: el de las instituciones grandes, generalmente en los países más desarrollados y el mundo de las instituciones más pequeñas, más numeroso, y que están en los países en vías de desarrollo. Ambos deberían ser complementarios pero en la práctica manifiestan las grandes brechas desintegradoras del sistema de la educación superior.

2) El segundo desafío emana del gran descuido de las IES con respecto al mundo profesional que habiendo egresado de sus ámbitos y cada vez más numeroso demanda formación continua y se constituye en una oportunidad muy importante de vinculación entre la universidad y la sociedad, la producción, el Estado, etc.

3) El tercer desafío es la construcción e implementación de áreas temáticas concretas de acción, espacios comunes de conocimiento y aprendizaje; áreas donde se construyen comunidades de aprendizaje, de socialización de información y programas de actualización profesional. Áreas de cooperación y colaboración en temas concretos. La ausencia de estas áreas es responsable de la ambigüedad y baja sostenibilidad de los esfuerzos colaborativos.

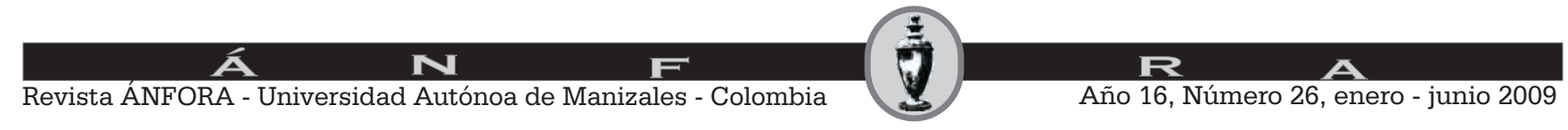




\section{Dos mundos}

El análisis de las redes de las instituciones de educación superior (IES) manifiesta que las instituciones grandes y de mayor prestigio han resuelto en gran medida los desafíos de calidad y han logrado en casi todos los países mejorar sus programas y sistemas administrativos. Estas instituciones cuentan con recursos para la investigación y han desarrollado un grado avanzado de cooperación con "partners" en otros continentes, especialmente Europa y Norteamérica. Por todo ello se han diferenciado del mundo predominante dentro de la región, el de las instituciones medianas, pequeñas, generalmente regionales y escasos recursos. Las instituciones que componen las redes mencionadas por el IESALC suelen contar con el apoyo de sus gobiernos y de mecanismos multilaterales de cooperación. Ello les garantiza recursos equivalentes a los objetivos que se proponen, lo que deriva en costos altos y estructuras administrativas grandes, burocráticas, que dificultan, al mismo tiempo, el cumplimiento de dichos objetivos. Todavía existen países en ALC, aunque son cada vez menos, cuyos gobiernos no apoyan financieramente la investigación, dependiendo ésta de los mecanismos de cooperación.

Muchas evaluaciones en el marco de los programas de cooperación interinstitucional particularmente entre la Unión Europea y América Latina y el Caribe (PIHE NETWORK 2007) así como de GUNI (2009) a nivel más global, constatan algunos hechos importantes: los grandes proyectos de cooperación entre las Instituciones de Educación Superior (IES) se ha orientado hacia la mejora de la calidad de los contenidos, instrumentos pedagógicos, sistemas administrativos y en menor medida a la investigación; en general se trata de programas relacionados con asuntos internos al sistema de la universidad. En muchos de estos proyectos ya se toma en cuenta a stakeholders como las instituciones de la comunidad, a los hacedores de políticas y a la propia cooperación. Pero todavía se trata de acciones puntuales sin continuidad, sin sistematización de las mismas.

Frente a las instituciones grandes, generalmente universidades públicas, se ha producido un crecimiento acelerado de universidades e instituciones de educación superior, lo que no ha significado necesariamente un aumento de la calidad de la oferta. Por el contrario la masificación de mala calidad y la ausencia de compromiso efectivo con las necesidades de la sociedad ha sido una característica de este fenómeno. Este crecimiento ha sido particularmente rápido y extenso en el sector privado profundizando en muchos casos la desarticulación de la enseñanza con la realidad.

Pero en este escenario dividido y marcado por las grandes brechas y donde prolifera la masificación de la educación de regular calidad, se puede también observar el surgimiento de iniciativas, no siempre procedentes de las grandes instituciones, que se replantean su relación con la sociedad, la comunidad, el Estado y sus gobiernos. Probablemente las organizaciones medianas y pequeñas viven más cerca del mundo real lo que sugiere la necesidad de que se

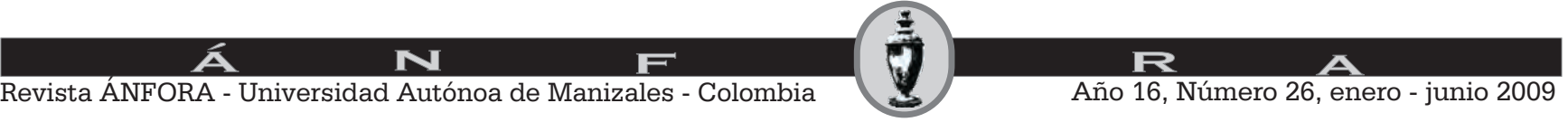


aborde en los estudios el conocimiento del potencial existente en este mundo de las instituciones pequeñas ya que representan parte del potencial existente en la región. En estas experiencias se otorga mayor importancia a los temas relacionados con el desarrollo del territorio.

En estos escenarios, las buenas prácticas están incluyendo en el concepto de calidad no sólo los aspectos internos al modelo y a la administración, al desempeño del aula, sino también criterios relacionados con la inclusión de temas "externos", relacionados con la pertinencia de su trabajo ante los problemas de la sociedad. Y no sólo para responder mejor al mercado de trabajo de los egresados, sino para incorporar la producción de un conocimiento más pertinente a la sociedad concreta y al momento global de la misma.

Algunas iniciativas, buscando un mayor compromiso entre la IES y la comunidad, han emanado de la propia sociedad, demandando a las instituciones de educación superior respuestas cualitativamente diferentes; otras veces las iniciativas han emergido de los propios estudiantes y muy pocas veces desde adentro de las propias universidades. En no pocos casos los gobiernos locales descentralizados convocan a las universidades e institutos en la elaboración de los planes de desarrollo. Sin embargo, en términos generales todavía persiste una gran brecha que distancia las instituciones de la sociedad y las de la universidad. Las redes de educación superior incorporan sólo parcialmente a las redes de desarrollo y a la inversa. De esta situación se deberá concluir la extraordinaria importancia de las experiencias en las que concurran ambas instituciones. Este hecho deberá ser considerado como un indicador de calidad.

Las brechas entre los dos mundos, cada día más profundas, marca el primer desafío: el acercamiento entre ambos mundos sobre la base del conocimiento del mundo real, buscando visibilidad para las instituciones más pequeñas y sus exitosas experiencias.

¿Son las instituciones pequeñas y aquellas de los países de menor desarrollo, el mundo real? Si y no. Pero por la cantidad y su masificación ameritan mayor atención. Por lo tanto un esfuerzo para hacer visibles las experiencias de las instituciones pequeñas agregará un gran valor al conjunto y puede servir para la formación de movimientos globales y que al mismo tiempo, por sus dimensiones son sostenibles.

Generalmente ese distanciamiento entre los dos mundos deja a una gran parte de los actores fuera de los beneficios de los avances en materia de la educación superior. La sistematización de las grandes tendencias al nivel global refleja parte de la situación real pero a veces no ayudan a identificar líneas de acción específicas. Muchas experiencias de procesos valiosos para la acción ocurren en las periferias, en países pequeños e instituciones hacia donde deberían enfocarse más la atención y las acciones.

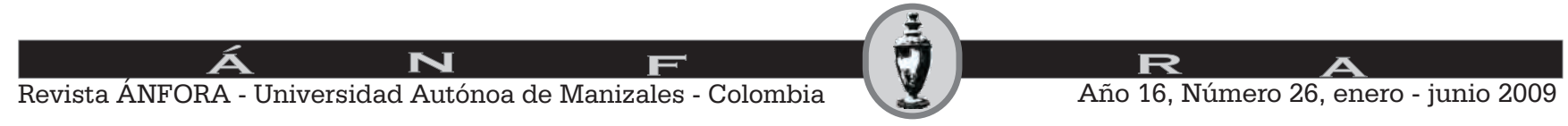


Las sistematizaciones globales deben alimentarse más con estas experiencias locales o pequeñas, lo cual evita que queden dispersas y sin una vinculación con los procesos globales. Por lo anterior, una de las metas debería ser el rescate de los aprendizajes basados en experiencias localizadas en diferentes países y tipos de IES y con "stakeholders" que requieren un proceso continuo de conocimiento. Entre las organizaciones pequeñas existe dinamismo y muchas iniciativas de aproximación a la sociedad que pueden enriquecer al resto.

\section{El Profesional como Vínculo con la Realidad y como Fuente de Conocimiento}

Un segundo desafío se refiere al mundo de los profesionales, quienes estando ya fuera del sistema universitario y de las instituciones de educación superior (IES), viven el día a día en la comunidad, en los gobiernos, en las empresas, ejecutando las políticas, los proyectos de desarrollo, y la cooperación internacional. En este gran desafío, los profesionales, los técnicos, los practicantes, los capacitadores y los funcionarios públicos, plantean hoy día la necesidad de una línea continua de comunicación con las fuentes de conocimiento, un sistema de formación continua y de actualización en su disciplina, y acercamientos interdisciplinarios al trabajo de implementación de los proyectos. Un distanciamiento entre las instituciones de educación superior y los profesionales implica el abandono de una fuente de conocimiento muy importante; el profesional es abandonado en la medida que no es un demandante de un programa de postgrado. Un acercamiento al profesional lo es a la realidad, ya que es el vínculo real con la misma.

Acercar al profesional a las IES representa, sobre todo en formatos interdisciplinarios, como en el caso del tema del desarrollo territorial, retornar a un sistema de aprendizaje donde se aprende haciendo y donde la acción está permanentemente informada por la investigación y las actividades de actualización. Los profesionales son los stakeholders más importantes de la educación superior, por lo que formación, investigación y difusión de conocimiento forman una gran unidad en la gestión del conocimiento para el cambio en un mundo global.

Las respuestas a los desafíos mencionados requieren de espacios institucionales adecuados y la construcción de puentes para que las experiencias pequeñas puedan ganar valor y agregarlo a los proyectos de las instituciones más grandes. Espacios de encuentro entre ambos mundos, actualmente separados, son posibles. En ellos académicos, profesionales y otros actores encuentran formas de vinculación continua con la producción del conocimiento. Es así como la asociación entre el mundo de la educación superior tendría mayores posibilidades de enraizarse en la comunidad.

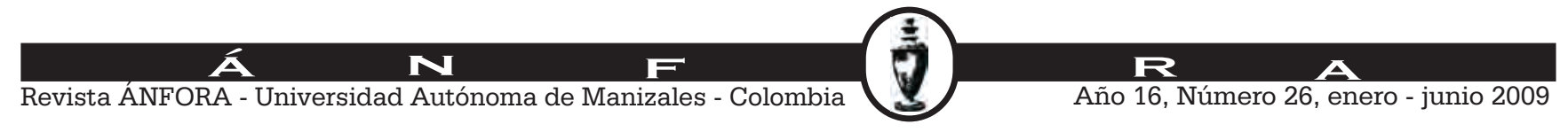




\section{Construcción de Espacios Concretos Comunes de Cooperación}

Una evaluación por hacer se refiere a las redes temáticas en las cuales se concretan estos diálogos entre la universidad y los profesionales, vinculados así mismo con sus comunidades, con el Estado, con las organizaciones de base.

Muchas de las redes agrupan a IES de un mismo tipo y en torno a programas puntuales; son escasos los ejemplos en que se observan como objetivo principal la investigación y en que, en torno al mismo, se organice la formación superior de los profesionales en áreas especificas, en espacios concretos de cooperación. Muchos de los mecanismos de cooperación internacional que apoyan la investigación prefieren proyectos para instituciones individuales y donde los objetivos sean fácilmente medibles sobre la base de documentos. Además, la mayoría de las redes adolece de los mismos problemas del sistema universitario: no siempre son respuestas al dialogo con la realidad, predominando las asociaciones corporativas, puntuales y espasmódicas. Se han realizado estudios sobre algunas de estas redes sobre todo de aquellas vinculadas con el desarrollo local que revelan, por un lado su importancia y por otro sus enormes limitaciones como respuestas a los problemas de la realidad. (GALLICCHIO, $2002 \mathrm{a} \mathrm{y} \mathrm{b)}$

La institucionalidad universitaria, es una hipótesis, podría ser una punto de apoyo importante para aportar a las redes continuidad, sistematicidad, metodología de trabajo y gestión del conocimiento, evaluación desde cierta distancia de la acción de los logros. Sin embargo, suelen producirse desencuentros en las instituciones universitarias con las exigencias de la vida real porque suelen ser estructuras pesadas y burocráticas, que difícilmente se suelen adaptar a las exigencias de los cambios y a la flexibilidad que exigen los tiempos de los sistemas de acción de la comunidad, para lo que las ONGs están mejor capacitadas. Pero como regla general, las ONGs no pueden permitirse el lujo de acción en investigación y formación programática por su alta dependencia de los organismos de financiamiento, lo que no soportan programas estratégicos.

Producir espacios concretos de cooperación entre ambos mundos es un gran desafío sobre el que se pueden empezar a observar algunas iniciativas, que son importantes en la perspectiva de que sirvan como espacios para estructurar programas y proyectos. ${ }^{6}$ Estos espacios que faciliten la continuidad y el seguimiento a los temas son algo ideal y utópico, pero que no por eso deberán desecharse.

\section{LA RIPPET Y LOS DESAFIOS PRESENTES.}

¿Cuáles serán los espacios donde RIPPET pueda ir encontrando su identidad y su razón de ser en un mundo globalizado? Se podría partir de la hipótesis de que ALC puede aportar mucho al mundo global, que en las instituciones
6 Destacan esfuerzos con relativa estabilidad en el tiempo: The Canadian Community Economic Development Network (CCEDNet) is a national member-led organization committed to strengthening Canadian communities by creating better economic o p portunities and enhancing environmental and social conditions. http://www.ccednet rcdec.ca/? q = en/home; REDESMA, La Red de Desarrollo Sostenible y $\mathrm{M}$ e d i $\mathrm{A}$ m bi e $\mathrm{nt}$ e ( REDESM A), proyecto impulsado por el CEBEM, trabaja desde 1999, con un servicio gratuito de difusión, una Revista Virtual y un portal informativo a usuarios de Latinoamérica y el resto del mundo. Participan en ella organismos universitarios, técnicos y expertos de proyectos locales y de cooperación, organizaciones de la sociedad civil. Su flujo informativo abarca a más de 150.000 receptores y trabaja en la formación de c o m u n id a d e s d e a p r e n d i z a je. Ve r http://www.redesma.org/ y en particular su programa favorito Red de Aprendizaje sobre conservación, http://rac.cebem.org/; Otro tipo de iniciativa es el proyecto Conocimiento, Cooperación y Desarrollo, donde se facilita el trabajo cooperativo en dialogo Norte - Sur en torno a cinco áreas temáticas, http://ccdckd.cebem.org/index_esp.p hp.

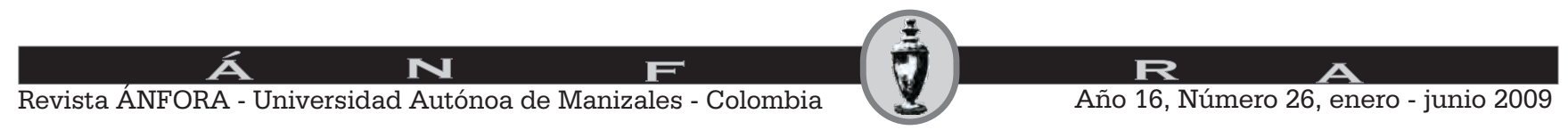


pequeñas y en los países de menor desarrollo relativo existen experiencias de gran valor para los aprendizajes globales. ¿Se puede pensar que es en estas instituciones pequeñas donde se puede experimentar con más probabilidad formas de relación entre la formación superior y la realidad, social, productora, etc.? (PABÓN Balderas Edgar A. 2009)

¿Cómo se sitúan las instituciones de la RIPPET en estos escenarios de problemas y desafíos? Los problemas de la red revelan con perfección todo lo mencionado. La apuesta será hacer ajustes y aprender del pasado para alcanzar formas de articulación de instituciones predominantemente pequeñas, que construyen experiencias novedosas, que se puedan visibilizar en la perspectiva de un movimiento. ¿Podría ser un aporte de ALC al movimiento mundial, a la globalización del compromiso entre la formación superior y los desafíos del desarrollo?.

La perspectiva de estas redes y plataformas de cooperación es pertinente hoy. La identidad y el futuro de la RIPPET es promisorio cuando, como nunca antes, la Red ha reunido en un encuentro internacional tantos participantes de ALC y, por primera vez también, a profesionales de importantes centros de educación superior y redes de Canadá. Finalmente hay que destacar el interés por parte de instituciones de educación, la sociedad regional y de los actores económicos.

Las instituciones de educación superior de la región ya cuentan con sistemas propios de acreditación; existen redes en la región y a nivel continental orientadas a ese objetivo La RIPPET ni podría ni debería competir con ellas, sino que, por el contrario deberá encontrar objetivos propios de valorización, visibilidad de las mejores prácticas. Se pueden movilizar recursos para generalizar un nuevo concepto de excelencia en los programas y en las formas de cooperación. Es sobre esta base que debemos analizar la naturaleza de la RIPPET y con base en ello buscar objetivos diferentes.

¿RIPPET es diferente o repite lo que se viene haciendo?, ¿Cuál es su misión principal? ¿Es que RIPPET es algo muy diferente de los otros tipos de redes ya existentes?

Una red de conocimiento que difunda las experiencias de colaboración y expanda modelos de cooperación que beneficie a los países menos desarrollados, puede servir para reducir, en parte al menos, las enormes brechas detectadas. En países pequeños y con pocos recursos se pueden rescatar importantes experiencias de mejoramiento de la educación superior y de su relación con la comunidad. Una red que coopere con otras agregando valor a sus propias actividades en el marco de un área de conocimiento donde concurren la investigación, la formación superior y la difusión de conocimiento.

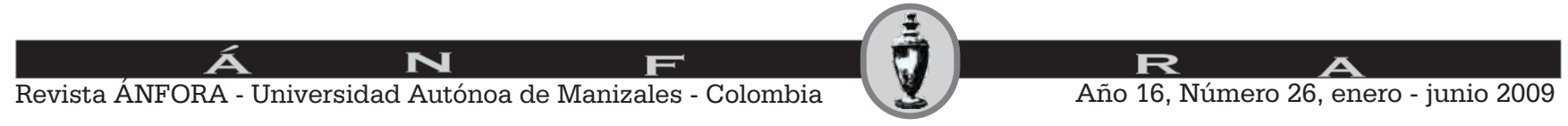




\section{Antecedentes de RIPPET}

Desde sus inicios la Red Iberoamericana de Investigadores sobre Globalización y Territorio (RII) generó una discusión interna sobre el tema de la formación superior y sobre la relación necesaria entre investigación y formación. Ya en sus inicios, en Río de Janeiro, 1996, se llegó a la conclusión de conformar un espacio para el intercambio y la cooperación entre los diferentes postgrados en Ibero América. El grupo de investigadores de la RII mostraba que la región contaba con valiosísimos recursos y se partía de la hipótesis de que coordinando actividades conjuntamente se puede agregar valor a las acciones individuales, tanto en formación como en investigación. A lo largo de los últimos 13 años, se trató de promover el intercambio, la coordinación y cooperación entre los distintos programas de postgrado que se realizan en el tema territorial en los países del Cono Sur, Brasil y México. Las experiencias de cooperación con Europa, han sido interesantes pero no se ha avanzado tanto. Aunque no se haya avanzado lo deseado y esperado, existen muchas evidencias de las posibilidades de dichas formas de cooperación.

En este contexto, se creó en Osorno, durante el V Encuentro, la Red Iberoamericana de Postgrados sobre Políticas y Estudios Territoriales (RIPPET), con el objetivo central de "intensificar la comunicación entre diversos Postgrados en el área urbano-regional" por medio del análisis y la discusión pormenorizada de las tendencias teórico metodológicas, las temáticas y los perfiles formativos de los programas sobre estudios territoriales. Posiblemente haya sido ese encuentro uno de los más importantes en la búsqueda de una identidad y donde se pone el énfasis en la continuidad del trabajo entre encuentros.

Una línea de trabajo importante puede orientarse a rescatar el valor de la diversidad de experiencias. Del análisis o anotaciones sobre las redes y desde la perspectiva del RIPPET, RII y RIER, interesa subrayar algunos aportes desde las Instituciones de Educación Superior a la reducción de las brechas que separa los dos mundos mencionados entre instituciones y entre países, y que en algunos casos dichas brechas se van profundizando.

La RIPPET ha caminado en dos direcciones de gran importancia para su futuro: el diálogo interinstitucional y el diálogo entre la investigación y la difusión de información. Existen razones para explicar por qué los avances en estas dos direcciones no han sido los deseados, aunque estamos seguros de que es el camino correcto. Ahora, en este VIII encuentro, interesa reflexionar y tomar decisiones para avanzar en esas dos direcciones, en un dialogo Norte - Sur y Sur-Sur, y estrechando los lazos de complementariedad entre la RII, la RIER y la RIPPET. Agregar a las líneas de acción pasadas, la coordinación entre las tres redes e incorporar el diálogo con IES del Canadá y redes mundiales, exige de una focalización más ajustada de los alcances y objetivos de la RIPPET. Posiblemente estemos ante un momento de inflexión por no decir fundacional

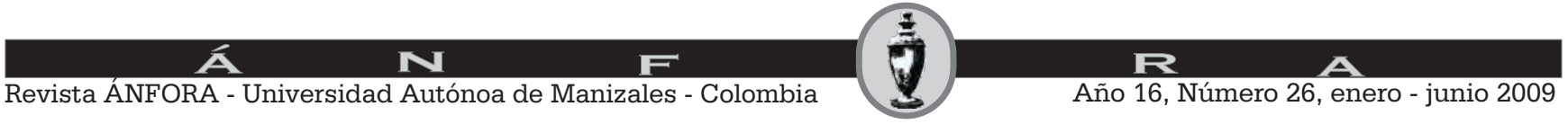


de una nueva forma de cooperación y colaboración más acorde a las exigencias del mundo actual.

Para ello es necesario avanzar en un diseño institucional creativo, flexible y arriesgado, por el que los objetivos y actividades cotidianas de las tres redes se encuentren de forma más continua en ese diálogo global, avanzando en el diálogo dentro de la región y con otras regiones de dentro y fuera del continente.

\section{Institucionalidad para la cooperación}

No arriesgamos mucho al decir que no tenemos clara la idea sobre el tipo de institución que se requiere para estos objetivos arriba mencionados. Cualquier propuesta que se haga deberá estar sometida a la prueba. La que se vaya construyendo tendrá que partir de la incertidumbre y no de formas y estructuras basadas en certidumbres, que suelen ser los principales criterios que buscan muchos colaboradores y sobre todo financiadores. Estos últimos no financian estas formas institucionales flexibles, por lo que es un asunto nuestro y depende de lo que nosotros queramos hacer con los proyectos que componen la RIPPET. ${ }^{7}$

Desde esta perspectiva la RIPPET se propone coordinar estrechamente con la RII y la RIER en sus actividades de investigación y en la tarea editorial. Así se fortalecerá la relación entre la formación, la investigación y la tarea editorial ser una de las bisagras entre la formación y la producción científica presentada en los encuentros de investigación. La coordinación es la forma de entender el tiempo en que vivimos, tiempo de producción colectiva de conocimiento, trae muchas ventajas a las tres redes, todas ellas relacionadas con el fortalecimiento de la colaboración.

Para no repetir lo ya existente ni competir con otras iniciativas en marcha en la región, y para lograr esta coordinación flexible se requiere de un diseño institucional adecuado que facilite de forma innovadora las actividades. Un diseño institucional como el de la RIPPET, basado en compromisos entre personas, radicadas en programas de instituciones, y sometido a la actualización continua, exige una coordinación que facilite la cooperación y los acuerdos entre los interesados. Este diseño le dará sentido a la RIPPET. Los encuentros, al igual que éste de Manizales, deben servir para visualizar y concretar espacios de interés común y propuestas de asociación y/o colaboración.

Para ello es importante vincular en torno a una misma estrategia de acción las actividades de las tres redes, que con el devenir del tiempo se fueron desarrollando de forma independiente debido a las particularidades de las mismas, a las actividades y a las responsabilidades institucionales con que las pusieron en marcha.
7 La RIPPET no asocia a instituciones sino a programas, por lo que su institucionalidad deberá ser flexible.

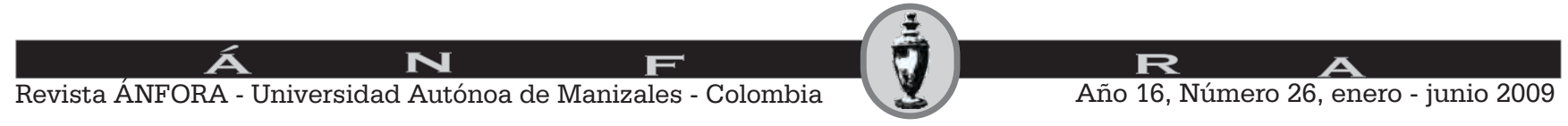


Es importante valorar el aporte institucional de cada una de ellas:

- La investigación fue impulsada por la Red Iberoamericana de Investigadores sobre Globalización y Territorio RII. En ello el Instituto de Estudios sobre la Universidad (IESU) de la Universidad Autónoma del Estado de México asumió en los últimos años las tareas de coordinación encabezadas por el Dr. Sergio González López.

http://www.uaemex.mx/pwww/rii/home.html

- Las actividades de docencia y formación surgieron desde su inicio en la reunión de Bahía Blanca, como una necesidad estrechamente relacionada con la investigación y que fue compartida por el Coordinador de la RII Sergio González y el apoyo de José Blanes, del Centro Boliviano de Estudios Multidisciplinarios CEBEM de Bolivia, actual Coordinador General de la Red Iberoamericana de Postgrados en Políticas y Estudios Territoriales RIPPET a partir del último encuentro en Río IV de Argentina. http://rippet.cebem.org/, con el apoyo del proyecto "cooperación Conocimiento y desarrollo.

http://ccd-ckd.cebem.org/index_esp.php

- La coordinación de la Red Iberoamericana de Editores de Revistas sobre temas de globalización y territorio RIER, Red Iberoamericana de Editores de Revistas sobre globalización y territorio a cargo Eduardo Aguado y Rosario Rogel de REDALyC, de la Universidad Autónoma del Estado de México a partir de la reunión de Bahía Blanca, y en particular del XI Encuentro llevado a cabo en Querétaro, se hacen cargo de su coordinación. www.redalyc.org.

Con esta coordinación se fortalecen las tres actividades de acuerdo a sus propias especificidades y llega el momento en que las mismas coordinen institucionalmente ya que se trata de una estrategia común: investigación, formación y comunicación.

Desde la perspectiva de la formación superior es un imperativo unir las tres actividades en torno a objetivos comunes o coincidentes, así como encontrar la estrecha relación que existe entre las tres. La formación profesional requiere de una permanente actividad de investigación y esta última deberá tener como primer objetivo la formación profesional. La coordinación de actividades de difusión, documentación y edición de revistas es un aspecto no sólo instrumental a las dos actividades anteriores, sino que se constituye en un área sustantiva, en un área de cooperación en la formación de comunidades que comparten actividades en un área temática. De hecho se pueden concretar comunidades científicas en las áreas temáticas de las otras dos redes. Mas aún, visto el desarrollo de la tarea de edición, se abren cambios temáticos nuevos y que facilitan la formación de comunidades profesionales para el dialogo Norte Sur y Sur - Sur.

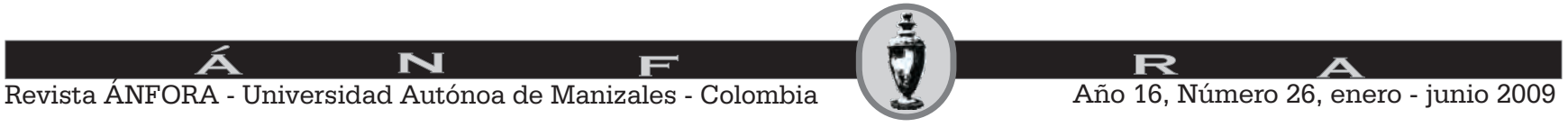


El gran desafío es lograr una forma institucional que facilite el trabajo propio de cada una de las redes, dar valor agregado a cada una de ellas, y al mismo tiempo una forma de coordinación que enriquezca el trabajo conjunto. Finalmente la coordinación de actividades de las tres redes es una forma de relacionar la investigación basada en la comunidad con el mundo de los profesionales. Al mismo tiempo esta coordinación puede servir para basar un proceso de formación continua.

La búsqueda de formas concretas para una estrecha coordinación de estas tres redes puede ser uno de los elementos identificadores y al mismo tiempo de diferenciación respecto de las redes existentes. El gran desafío pendiente es el de lograr la sostenibilidad del aporte institucional de las instituciones que sirven de soporte a las tres redes. Un segundo desafío consiste en desarrollar todas las dimensiones de trabajo en torno al área específica del desarrollo del territorio desde la perspectiva de la globalización. Aunque el tema territorial es un área temática concreta, para la construcción de un espacio común, todavía se requieren de mayores precisiones en torno a sub áreas, como se expresa en los grupos de trabajo de la RII o en las posibles comunidades que pueden emanar de la base de Información de REDALyC

\section{Una tarea central: la visibilización de los programas}

Es importante una institucionalidad, en el caso de RIPPET, que le dé alta visibilidad dentro y fuera de la región, que garantice y además manifieste su valor y seriedad. La piedra fundamental de RIPPET es su capacidad para dar valor agregado a la calidad de los cursos y programas de formación que se ofrecen por parte de las instituciones de la región. Muchas instituciones podrían apoyarse en RIPPET para incursionar en diferentes niveles o formas de cooperación y colaboración que, por su naturaleza, llegan a todos los países de la región y fuera de ella.

De manera similar a las otras dos redes, la de RIPPET es una forma flexible de institución, no cuenta con una personería jurídica, lo que hace más fácil y flexible su funcionamiento. Las tres redes se apoyan en la responsabilidad y colaboración de sus miembros, y en el apoyo de una institución que la acoge y la impulsa. Se ha discutido muchas veces sobre esta forma de institución de libre asociación, de corresponsabilidad y de compromiso por parte de una institución, y se concluyó que sus ventajas compensan enormemente sus limitaciones. Se apoyan en infraestructuras existentes, lo que implica un enorme ahorro de recursos y facilita la sostenibilidad; no requieren de un staff dedicado a la búsqueda de financiamientos para mantener una burocracia que muchas veces impone su propia lógica a la vida de las redes.

El concepto de institucionalización se refiere más a los aspectos funcionales que formales. No se trata de crear estatutos y reglamentos para una personaría jurídica. Esta institucionalización se basa en la aceptación por parte de sus

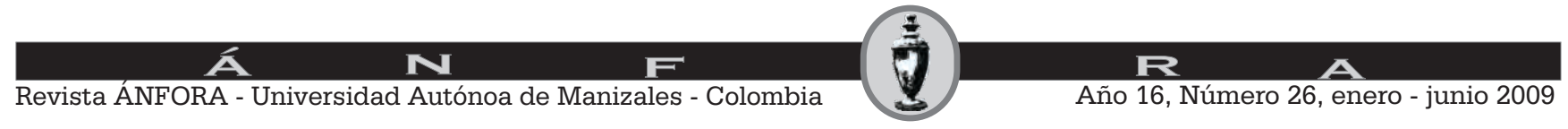


miembros de acuerdos comunes y de una estructura de funciones que se generan y que se ejecutan mediante miembros de la RIPPET y otros que se adhieran a ella. RIPPET se visibiliza como una institución que se sustenta en los acuerdos de consenso de sus miembros y en los recursos de cada uno de ellos. Un grupo de instituciones conforman diferentes mecanismos de coordinación y le dan visibilidad y sostenibilidad a la RIPPET.

Un aspecto que merece resaltarse por su gran importancia es la responsabilidad asumida por las instituciones que asumen el peso financiero y logístico para la realización de los encuentros internacionales. En los ocho encuentros se ha invertido una gran cantidad de recursos locales y un enorme despliegue institucional para que investigadores, docentes y miembros de redes de la sociedad civil puedan encontrarse.

Sin duda que los problemas de coordinación entre instituciones, que tienen por su lado cada una sus propios objetivos y actividades, limita mucho el desarrollo de actividades en los periodos entre encuentros. Pero compensa la riqueza de una "membresía" voluntaria y funcional a la situación de cada uno en el tiempo de sus propias instituciones.

\section{HACIA UNA PROYECCIÓN}

¿Cuales podrían ser los pasos siguientes que deberemos proponernos en este Encuentro? ¿Con que nos vamos a volver cada uno de nosotros después de Manizales?

- Descubrir las formas de valorización de programas y de criterios no habituales en la región, a lo que habrá que dar alta visibilidad.

- Avanzar hacia formas y modelos de cooperación como aporte nuestro a la región.

- Encontrar espacios de responsabilidad en este tarea.

- Las actividades que identifiquemos deberán servir para crear un espacio de cooperación, para lo que nos propondremos: formación de comunidades concretas de cooperación; construcción de espacios de información y comunicación; desarrollo de cursos en línea de forma colaborativa y

- Los mecanismos de difusión y la organización de cursos cortos y otras actividades como la Revista y los cuadernos de clase, serán medios muy importantes para que la RIPPET esté presente en el continente.

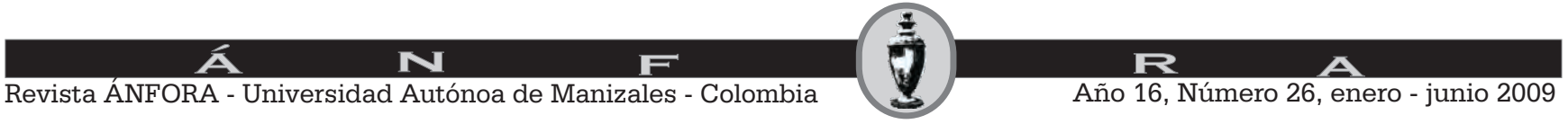


La partición de los miembros en encuentros internacionales serán oportunidades muy importantes para al visibilidad de la RIPPET fuera del continente.

\section{La valorización de los programas que se ejecutan en el marco de la misma}

La imagen de la RIPPET es la de una red de profesionales académicos e instituciones que desarrollan programas de postgrados en temas territoriales, caracterizados por su calidad y por su orientación a la colaboración entre instituciones. En la medida de lo posible se espera que en algún momento se pueda contar con un programa regional.

Se trata de crear y hacer visible un mecanismo funcional que da valor a los programas de la RIPPET. Al no ser un mecanismo de acreditación se concentra en otro papel, el de dar valor a los programas introduciendo valores cualitativos como los mencionados. Sin restar importancia a las tareas de validar, acreditar, certificar, etc., la valorización de los programas adquiere una dimensión de especial importancia, más allá de los aspectos formales. Los programas se ven librados a la búsqueda de valores nuevos, en permanente definición y adecuación a las condiciones del país, la región y de los diferentes stakeholders. No se le puede pedir a RIPPET lo que no se propone, pero lo que si puede hacer la RIPPET es introducir en el mundo de las IES nuevos valores, más allá de los formales, de acreditación académica. Este puede ser su papel central, incidir en el sistema de valores, incorporando en un nuevo concepto de calidad la colaboración como aporte central, su orientación a los profesionales y la configuración de comunidades de aprendizaje.

Esto tiene múltiples implicaciones: la primera es que la RIPPET agrega a las prácticas institucionales ya establecidas de supervisión, otros parámetros cualitativos. Entre esos parámetros sea el de la cooperación y el compromiso con la sociedad, el del diálogo entre nosotros y con la realidad local. No son temas asumidos por la certificación que mira más al aula y los contenidos. Calidad en términos de la RIPPET es un añadido de enorme importancia, el compromiso y la producción colectiva de conocimiento. Es lo que la RIPPET puede aportar en valor a los programas: la colaboración y trabajo en red.

En este sentido, planteamientos como los de los siguientes adquieren mayor consistencia. Es hoy día, en la región y en un diálogo interinstitucional, un valor de los programas:

- Generar actividades que favorecen el intercambio y la cooperación entre los miembros

- Agregarle un valor adicional a los programas reconocidos por ella, mediante el reconocimiento de la calidad académica del programa de postgrado con base en criterios, entre otros a discutir, como:

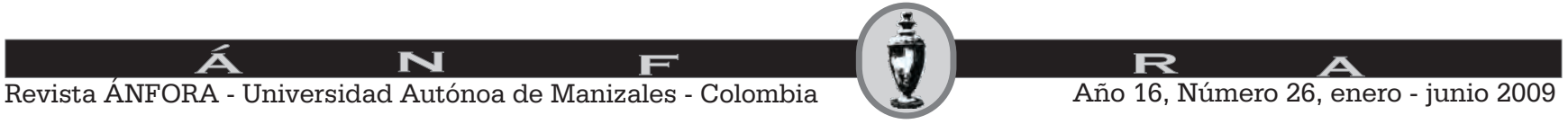


- cooperación regional y transnacional

- intercambio de profesores

- fomentar colaboraciones entre programas sean nacionales o internacionales

- desarrollar proyectos de ámbito regional

- relación con la investigación

- vincular la academia con la realidad

Dar visibilidad a estos aspectos es uno de los ejes centrales de la RIPPET. Hacer que los programas de la RIPPET, no sólo sean vistos como los mejores en sus países, gracias a los valores anteriores, sino que también lo sean en la región por una serie de cualidades meritorias bien reconocidas como las mencionadas. Si las dimensiones mencionadas son consideradas valores actuales en los procesos de producción y gestión del conocimiento comprometido con la realidad, ya merece la pena la construcción de la RIPPET:

- Desarrollar una política de visibilidad a estos programas por los valores mencionados en torno a la colaboración y la cooperación.

- Que sean programas reconocidos en al región más allá de los valiosos procesos de acreditación académica de cada país y de los sistemas de redes.

- Desarrollar y actualizar los mecanismos de comunicación más pertinentes para la visibilidad de las actividades de la RIPPET constituyen un aspecto central en torno al cual concitar la cooperación.

El aporte de la RIPPET al diálogo global, contribuyendo a ello con la visión desde el Sur es un criterio de valoración central. En sus áreas temáticas el aporte de ALC es fundamental, sobre todo como un contrapunto a mundos donde predominan planteamientos académicos muy generales, sistemas más corporativos y burocratizados.

El tema del desarrollo territorial se ha colocado como uno de los principales temas de interés en el mundo global y en la producción y gestión del conocimiento. Ante la escasa alimentación desde el mundo académico a las diferentes áreas relacionadas con lo territorial, la RIPPET pueda convertirse en un espacio para facilitar el diálogo entre redes consolidando este área en la región.

RIPPET debe prepararse de forma colaborativa para el diálogo con otras realidades y para que ello no ocurra de forma esporádica y sobre la base de impulsos que ocurren cada que se realiza un encuentro; los encuentros internacionales deberán avanzar en una dirección precisa hacia la identificación y consolidación de compromisos de trabajo conjunto entre instituciones.

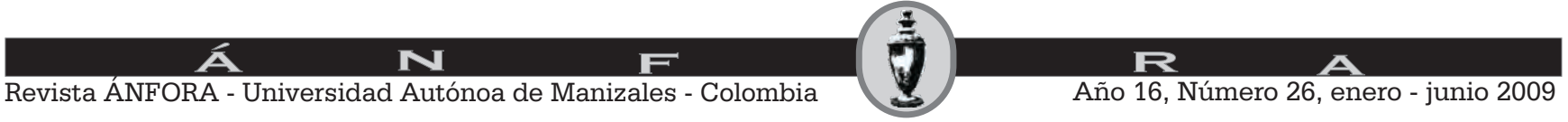


Este encuentro ha sido un compromiso para seguir investigando y aprendiendo formas de diálogo con el profesional que día a día trabaja en soluciones o en gestión en políticas y proyectos. Un buen ejemplo de ello es trabajo de construcción Universidad Sociedad Empresa encontrado en el Eje Cafetero y sus instituciones.

El tema del desarrollo territorial se ha colocado como uno de los principales temas de interés y en el que hemos detectado una gran demanda de actualización profesional como se muestra en el tema desarrollado por Pabón. Este tema se verá reforzado por la colaboración de la Redalyc con instituciones y programas de la RIPPET.

- La cooperación transnacional se puede hacer realidad mediante acuerdos con programas como los que se han presentado en el Encuentro, y en torno a actividades de actualización profesional. En ello colaborará el programa "Cooperación Conocimiento y Desarrollo que impulsan el CEBEM y seis instituciones canadienses".

- En el Encuentro de Manizales se han abierto nuevas oportunidades de cooperación con instituciones de otros países como Brasil, Ecuador, Perú. Esta relación seguirá en expansión.

- Para ello una relación con Global Alliance es muy importante y le dará a RIPPET una visibilidad en Asia, África y Europa.

Toda la gama de recursos de profesionalización con que cuentan las instituciones y programas son el capital de RIPPET y deberían contar con espacios de actualización a los profesionales que nunca tendrán la oportunidad de regresar al aula.

Consolidando un espacio de encuentro, el portal de la RIPPET deberá reflejar y vincular a los sitios de todos los programas, instituciones y redes. No se tratará de un portal más sino el espacio construido de forma conjunta.

Este espacio se dotará de todos los mecanismos para la visualización y el intercambio a través de:

1- Cooperación en un área temática, contribuyendo con información sobre programas, profesionales y actividades institucionales.

2- Elaboración conjunta de cursos de actualización para construir un espacio de cooperación y diálogo.

3- Dar visibilidad a nuestros logros a través de cursos, foros y el uso de las TICs, boletines y revistas, bases de datos y espacios virtuales interactivos.

Los encuentros de la RIPPET deberán ser de encuentro para conocerse, negociar y armar propuestas entre instituciones y/o personas.

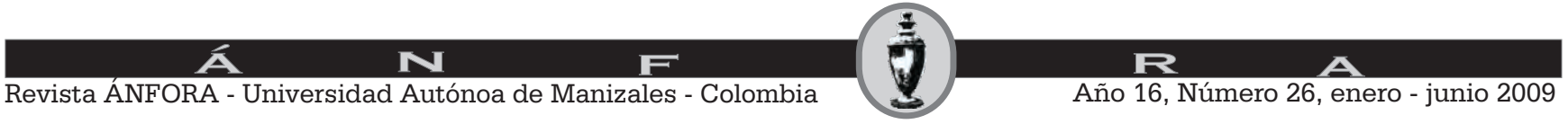




\section{Referencias}

BLANES, José, 2008. Desarrollo local y comunitario. Múltiples rutas para las comunidades de aprendizaje. Local and communitarian development. Multiple pathways for learning communities. Développement local et communautaire. Des multiples voies pour les communautés d'apprentissage. CEBEM, La Paz.

http://osgeydel.cebem.org/docs/osgeydel_es.pdf

BRUNNER, J. J. (2005) Tendencias recientes de la educación superior a nivel internacional. http://mt.educarchile.cl

GALLICCHIO Enrique, 2002 a La construcción del desarrollo local en América Latina, Análisis de experiencias. Building local development in Latin America

Experiences' análisis. Programa Alianzas Estratégicas para el Desarrollo Local en América Latina Program «Strategic Alliances for Local Development in Latin America». Centro Latinoamericano de Economía Humana (CLAEH), Asociación Latinoamericana de Organizaciones de Promoción (ALOP) Montevideo

GACER, Global Alliance on Community Engaged Research. 2009 Educación Superior. Compromiso Comunitario y el Mundo Que Queremos. Resumen de políticas para la Conferencia Mundial sobre Educación Superior, 5 al 8 de julio de 2009. http://communityresearchcanada.ca/

GALLICCHIO Enrique, Ioanna GROTIUZ y Matilde SUÁREZ 2002 b. Redes Internacionales de Desarrollo Local en América Latina. Programa de Desarrollo Local - Centro Latinoamericano de Economía Humana (CLAEH). Montevideo, Uruguay.

GIL, Luciana y OBAYA, Martín, Las redes universitarias en América Latina y el Caribe (primer borrador presentado a miembros Vertebralcue, abril 2009)

Universidad de Bologna, Representación en Buenos Aires.

GUNI, Global University Network for Innovation, 2009, : Educación superior en tiempos de cambio: Nuevas dinámicas para la responsabilidad social. Nuevas dinamicas para la responsabilidad social. Madrid. Barcelona. México. Ediciones Mundi Prensa, Madrid, 2009.

PABÓN Balderas Edgar A. 2009 "Cursos cortos y experiencias de cooperación en postgrados conjuntos". CEBEM. Bolivia. VII Encuentro de Postgrados Iberoamericanos sobre Desarrollo Regional y Políticas Territoriales, RIPPET, Universidad Autónoma de Manizales, Manizales - Colombia 5-8 de Agosto de 2009.

PIHE NETWORK, 2007, prácticas y tendencias para la internacionalización y la cooperación entre universidades de América Latina y Unión Europea.

SEGRERA, Francisco López, 2009 Educación superior internacional comparada (Escenarios, Temas y Problemas) Proyecto Vertebralcue, UNIBO, Buenos Aires.

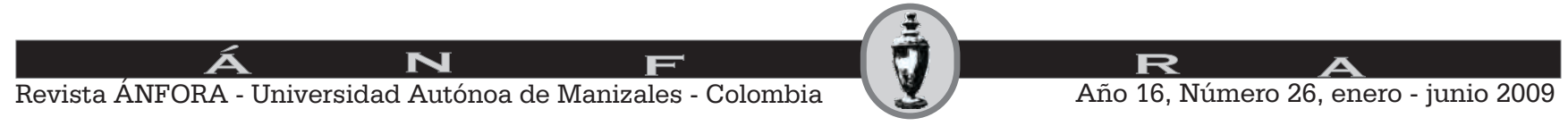

